Brit. Heart J., 1965, 27, 896.

\title{
SIGNIFICANCE OF RETROGRADE CONDUCTION IN A-V DISSOCIATION
}

\author{
BY \\ L. SCHAMROTH AND H. D. FRIEDBERG \\ From the Baragwanath Hospital and University of the Witwatersrand, Johannesburg, S. Africa
}

Received January 4, 1965

In certain circumstances the discharge rate of the A-V node may exceed that of the S-A node. The A-V node then becomes the dominant pacemaker of the heart. If, in addition, there is retrograde unidirectional A-V block, the atria remain under control of the S-A node, and A-V dissociation results. When a sinus impulse reaches the A-V node during the non-refractory phase, it is conducted to, and captures control of, the ventricles for one beat-known as a capture beat; occasionally 2 , or rarely 3 , consecutive capture beats occur. An analogous situation may arise when the ectopic pacemaker is situated in the ventricles.

This communication reports further observations on $\mathrm{A}-\mathrm{V}$ nodal conduction in $\mathrm{A}-\mathrm{V}$ dissociation, with special reference to conduction in consecutive capture beats.

\section{A-V Dissociation with Paired Consecutive Capture Beats}

Case 1. The electrocardiogram (Fig. 1) shows dissociation between sinus and A-V nodal rhythms. The sinus cycle is regular at 112*; the A-V nodal cycle is regular at 110 . The first 4 QRS complexes are A-V nodal beats. The next 2 QRS complexes (labelled R3 and R4) are premature and represent consecutive ventricular captures. The next QRS complex is slightly premature (106) and probably represents a third consecutive ventricular capture. The P-R interval of the first capture beat is 76; that of the second 25 . The $R-R$ interval terminated by the first capture beat-the capture interval-is 86 ; the second capture interval is 62 .

Case 2. The electrocardiogram (Fig. 2) shows dissociation between rapid sinus and A-V nodal rhythms. The sinus cycle is regular at 52; the A-V nodal cycle is regular at 47 . The first 4 QRS complexes represent A-V nodal beats; the next 2 QRS complexes (labelled R3 and R4) are premature and represent consecutive ventricular captures. The $P-R$ interval of the first capture beat is 34; that of the second 22 . The first capture interval is 42 ; the second 37.

Case 3. The electrocardiogram (Fig. 3) shows dissociation between sinus and A-V nodal rhythmssinus and A-V nodal tachycardia. The sinus cycle is regular at 56 ; the A-V nodal cycle is regular at 48. The first 3 QRS complexes are A-V nodal beats; the next 2 QRS complexes (labelled R3 and R4) are premature and represent consecutive ventricular captures. The P-R interval of the first capture beat is 30; that of the second 16. The first capture interval is 34; the second 42. The first capture beat is conducted with slight aberration.

Case 4. The electrocardiogram (Fig. 4) shows dissociation between sinus and A-V nodal rhythmssinus and A-V nodal tachycardia. The sinus cycle is regular at 52; the A-V nodal cycle is regular at 50. The first 4 QRS complexes are A-V nodal beats; the next 2 QRS complexes (labelled R3 and R4) are premature and represent consecutive ventricular captures. The P-R interval of the first capture beat is 23; that of the second 16. The first capture interval is 37; the second 43. The first capture beat is conducted with aberration.

* All time intervals represent hundredths of a second, e.g. $62=62$ hundredths of a second. 


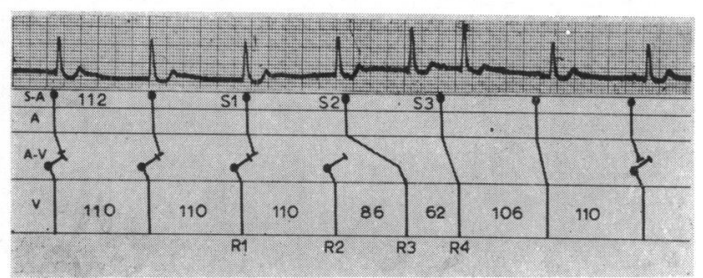

FIG. 1.-Case 1. Electrocardiogram showing consecutive capture beats during dissociation between sinus and A-V nodal pacemakers.

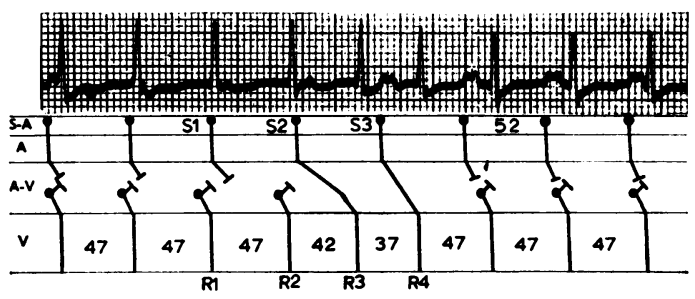

FIG. 2.-Case 2. Electrocardiogram showing consecutive capture beats during dissociation between sinus and A-V nodal pacemakers.

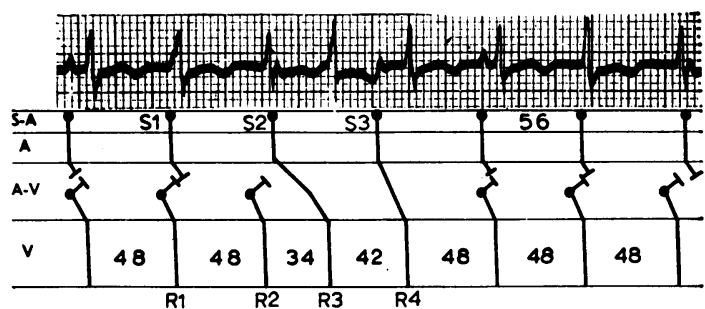

Fig. 3.-Case 3. Electrocardiogram showing consecutive capture beats during dissociation between sinus and A-V nodal pacemakers.

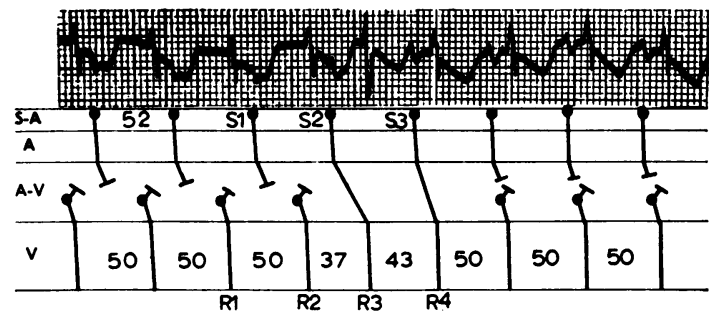

FIG. 4.-Case 4. Electrocardiogram showing consecutive capture beats during dissociation between sinus and A-V nodal pacemakers.

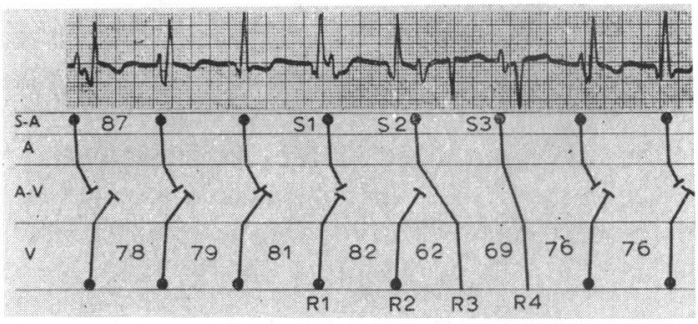

FIG. 5.-Case 5. Electrocardiogram showing consecutive capture beats during dissociation between sinus and ectopic ventricular pacemakers.

Case 5. The electrocardiogram (Fig. 5) shows dissociation between sinus and ventricular pacemakers. The sinus cycle is regular at 87; the ventricular cycle is regular at \pm 79 . The first 5 bizarre QRS complexes represent ventricular beats. The next 2 QRS complexes (labelled R3 and R4) are premature and normal in shape and represent consecutive ventricular captures. The P-R interval of the first capture beat is 34 ; that of the second 18. The first capture interval is 62 ; the second 76 .

\section{Discussion}

The $\mathbf{P}-\mathbf{R}$ interval of the first of paired ventricular captures is longer than that of the second. This is because the first capturing impulse is more premature than the second and consequently traverses tissues that are relatively more refractory. In Case 4, the P-R interval of the first capture beat (23) is somewhat longer than that of the second. However, the P-R interval of the first capture beat and the resultant capture interval are often inordinately long in comparison to those of the second, i.e. longer than would be expected from prima facie consideration of the state of recovery of the A-V conducting tissues. Thus, in Case 1, the P-R interval of the first capture beat is over 3 times as long as its successor; and the second capture interval (62) is shorter than the first (86). Similarly, 

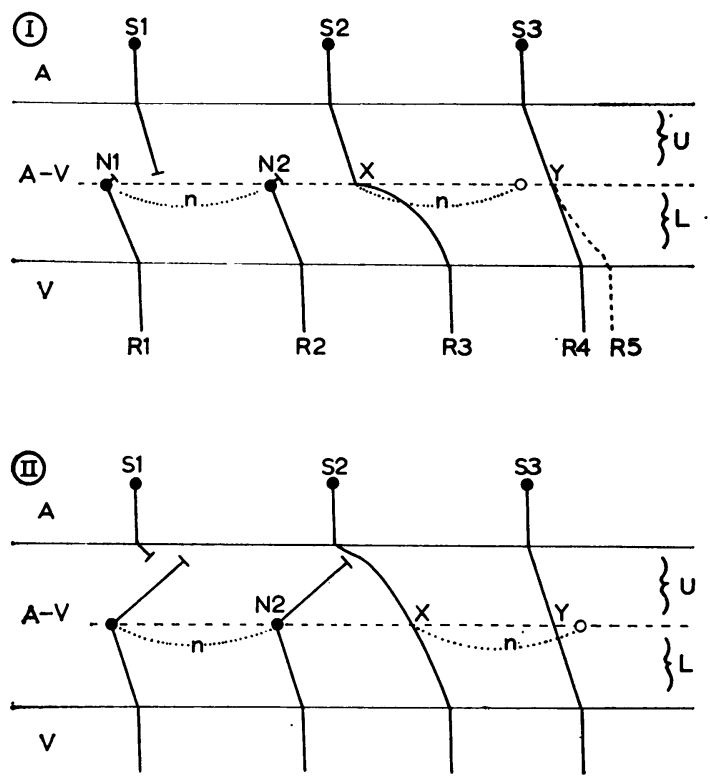

Fig. 6.-Diagrammatic representation of conduction mechanisms in consecutive capture beats. See text. the long $\mathbf{P}-\mathrm{R}$ interval of the first capture beat in Case 2 leads to a capture interval that is longer than the second. It is impossible to explain these time relationships in terms of simple anterograde delay alone. Mechanisms which may operate in this situation are considered below.

Conduction Mechanisms in Paired Capture Beats. In dissociation between sinus and A-V nodal pacemakers there are 2 parts of the A-V node where the sinus impulse of a capture beat may be delayed: in the upper part of the node, i.e. above the level of the A-V nodal pacemaker (illustrated as zone $U$ in Fig. 6); and in the lower part of the node, i.e. below the pacemaker (illustrated as zone L in Fig. 6). Delay may occur in either, or in both, zones.

Delay in the lower zone only. If there is no retrograde penetration of the $A-V$ nodal impulses, both capturing impulses ( $\mathrm{S} 2$ and $\mathrm{S} 3$ in Diagram I of Fig. 6) will find the upper zone (zone $U$ ) fully recovered and will, there-

fore, be conducted through this zone at the same rate. The sinus interval is, therefore, maintained to the level of the nodal pacemaker, i.e. the interval S2-S3 is equal to the interval X-Y of Fig. 6. Below this level, however, the sinus interval will not be maintained. In the uppermost part of zone $\mathrm{L}$, the sinus impulse of the first capture beat (S2) is more premature in relation to its preceding impulse than the sinus beat of the second capture (S3) is in relation to the impulse that preceded it, i.e. the interval N2-X is less than the interval X-Y in Diagram I of Fig. 6. Since the conducting tissue encountered by the first sinus impulse has had less time to recover, the first sinus impulse will here be conducted more slowly than the second. However, in the lowermost part of zone $\mathrm{L}$, the second sinus impulse is the more premature impulse as shown by the respective $R-R$ intervals, i.e. R2-R3 is greater than R3-R4. Thus, in the lowermost part of zone $L$, the second sinus impulse must be conducted more slowly than the first. In other words, the first capturing sinus impulse, initially greatly slowed, must meet progressively less refractory tissue and should then accelerate; while the second sinus impulse, initially conducted quickly, must meet increasingly refractory tissue and should be slowed. The line representing the passage of the first sinus impulse will not be straight but a curve (Diagram I of Fig. 6); the shape of this curve affects the shape of the second sinus impulse, which should resemble the dotted line Y-R5 in Diagram I of Fig. 6. This impulse should reach the ventricles, not at point R4, but at some point $R 5$ to give $\mathbf{P}-\mathbf{R}$ and $\mathbf{R}-\mathbf{R}$ intervals more comparable with those of the first capture beat. Under such circumstances, the second capture interval cannot be less than the first capture interval.

This phenomenon may also be viewed in terms of the conventional recovery curve. When the relationship of $P$ waves to $Q R S$ complexes varies, a recovery curve can be constructed by plotting each R-P interval (along the abscissa or $\mathbf{X}$ axis) against its respective $\mathbf{P}-\mathbf{R}$ interval (up the ordinate or $\mathrm{Y}$ axis). Such recovery curves are usually slightly convex upwards. Recent work, however, has shown that the curve is practically linear and forms an angle not exceeding $\mathbf{4 5}$ degrees with the $\mathrm{X}$ axis (Jedlicka, 1960). Even if the recovery locus is slightly curved, a straight line joining the co-ordinates of the shortest and longest R-P intervals cannot exceed an angle of 45 degrees with the $\mathrm{X}$ axis (this line will be referred to as the recovery line). This principle is illustrated in Fig. 7. Diagram I illustrates the situation when the recovery line forms an angle of less than 45 degrees with 
(I)

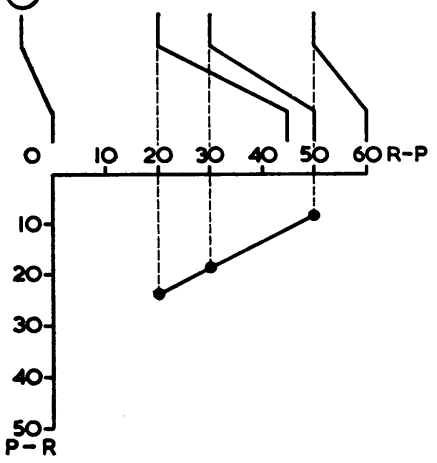

(1)

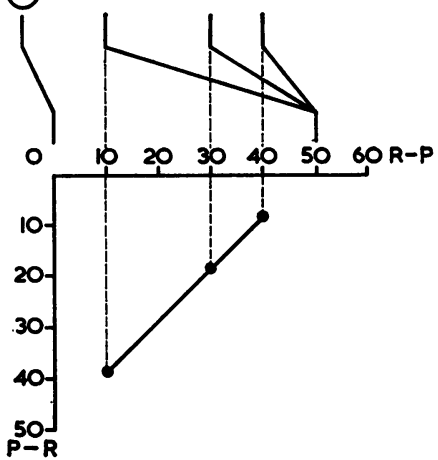

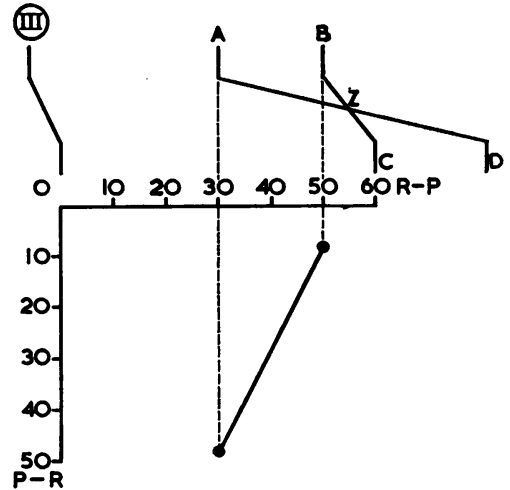

Fig. 7.-Diagrammatic representation showing various theoretical recovery lines formed by premature impulses. The first conduction line situated above the zero co-ordinates represents the preceding beat. See text.

the $\mathrm{X}$ axis; the latest, or least premature, impulse gives rise to the longest $R-R$ interval. Diagram II illustrates the situation when the recovery line forms an exact angle of 45 degrees with the $X$ axis; under this circumstance, all premature impulses give rise to equal $\mathbf{R}-\mathbf{R}$ intervals. Diagram III illustrates the theoretical situation should the recovery line form an angle greater than 45 degrees with the $\mathrm{X}$ axis; under this circumstance, an early impulse (A) would result in an anomalously long $\mathbf{P}-\mathbf{R}$, and consequent $\mathbf{R}-\mathbf{R}$, interval, i.e. it gives rise toa longer $R-R$ interval than a laterimpulse. Thus, the early impulse $A$ is conducted with a P-R interval $A-D$; a later impulse $B$ is conducted with a $P-R$ interval B-C. However, the lines representing the $P-R$ intervals must cross at point $Z$. It is clear that at point $Z$ the recovery of the A-V conducting tissues must be the same for both impulses. These impulses must thereafter be conducted at the same rate and reach the ventricles at the same time, i.e. impulse A cannot reach the ventricles later than $\mathrm{C}$. This is precisely the situation that arises if it is postulated that only delay due to previous anterograde conduction affects consecutive captures. The angles formed by the recovery lines

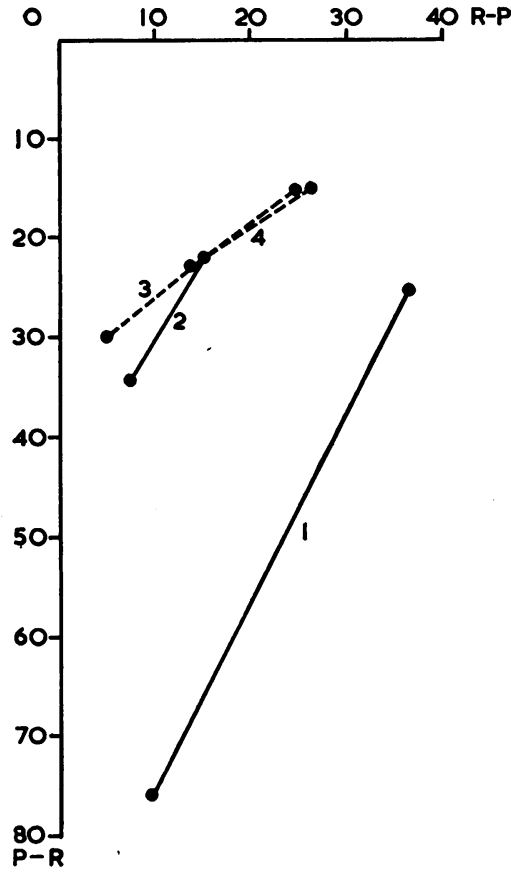

FIG. 8.-Graph illustrating recovery lines derived from the conduction of capture beats in Cases 1 to 4 . and the $X$ axis of the first 4 cases are 62, 59, 32, and 29 degrees respectively (Fig. 8)*. The recovery

* Note: It is not necessary in any given case to determine the angle the recovery line would make with the $\mathrm{X}$ axis. A modification of the point-slope equation of a straight line allows the slope to be calculated by the following simple formula:

e.g. Case 4:

$$
\frac{\mathrm{P}-\mathrm{R}(\mathrm{Max})-\mathrm{P}-\mathrm{R}(\mathrm{Min})}{\mathrm{R}-\mathrm{P}(\mathrm{Max})-\mathrm{R}-\mathrm{P}(\mathrm{Min})}=\tan a \text { (the trigonometric tangent of the required angle), }
$$

$$
\frac{23-16}{27-14}=\frac{7}{13}=0.55 \text {. }
$$

The resultant tangent cannot exceed unity for it would then form an angle greater than 45 degrees. Application of this formula to the first 4 cases reveals tangents of $1 \cdot 89,1 \cdot 7,0 \cdot 63$, and $0 \cdot 55$, respectively. The first 2 cases thus have anomalous tangents of greater than 1 . 
lines of the first 2 cases thus form angles greater than 45 degrees with the $X$ axis; and superimposition of the conduction lines of the first and second captures in these cases results in anomalous cross-over points similar to that found in Diagram III of Fig. 7.

Thus in the lower zone a similar delaying factor affects both the first and second capture beats, i.e. partial refractoriness resulting from preceding anterograde conduction. As this alone cannot satisfactorily explain the anomalous situation in Cases 1 and 2, other factors must be sought which would affect the passage of the first capturing impulse to a much greater extent than that of the second. The only possible factor which has a direct effect on the first capture beat but none whatsoever on the second is retrograde conduction of the preceding nodal impulse. This concealed retrograde conduction of the nodal impulse will cause refractoriness in the upper nodal zone thereby affecting the subsequent conduction of the first capturing impulse.

Delay both above and below the level of the A-V nodal pacemaker. Diagram II of Fig. 6 illustrates the effects of partial retrograde penetration of the A-V nodal impulse. The sinus beat of the first capture beat is very delayed in the upper zone of the node (zone $U$ ), because it meets partially refractory tissue; this is due to the fact that this zone has been traversed shortly before by the retrograde nodal impulse - N2. The sinus impulse of the second capture beat suffers no such delay in zone $U$ because it follows a sinus and not a nodal impulse. Thus the sinus interval is not maintained at the level of the nodal pacemaker, i.e. the interval X-Y is less than the interval S2-S3. The marked delay of the first impulse in the upper zone causes its QRS complex to be shifted towards the QRS complex of the second capture beat thereby contributing towards the relatively short second capture interval. Both sinus impulses may be further delayed below this level but the difference in delay will not be marked. If, therefore, there is partial retrograde penetration of the A-V nodal impulse, conditions that delay the conduction of the first capturing impulse in the upper part of the node will not apply to the second or later capturing impulses.

These principles are substantiated by the conduction mechanism of paired capture beats in dissociation between sinus and ventricular pacemakers (Fig. 5). In these circumstances it is clear that the delay of the first sinus impulse in the A-V node can only be due to refractoriness resulting from retrograde penetration of the preceding ventricular impulse.

Thus, when the concept of concealed retrograde penetration by a nodal or ventricular impulse is invoked, the inconsistency in time relationships is resolved. This possible effect of concealed retrograde penetration has received insufficient emphasis in the past. Recently, Moe, Abildskov, and Mendez (1964) have shown experimentally that ectopic impulses regularly penetrate the A-V nodal conducting tissues to a greater or lesser extent. Such retrograde penetration must have been operative in Cases 1, 2, and 5; and was probably operative in Cases 3 and 4.

\section{Mechanisms Allowing Consecutive Captures}

If the rates of both pacemakers of a dissociated rhythm remain constant, consecutive captures cannot occur. The first capturing impulse prematurely discharges the faster ectopic pacemaker, whose next spontaneous discharge will occur before the following sinus impulse could reach it. This is illustrated in Diagram I of Fig. 6. The nodal interval (labelled $n$ ) is shorter than the sinus interval. The nodal pacemaker is prematurely discharged at $X$ by the first capturing impulse (S2) and its next spontaneous discharge should follow $X$ after one nodal interval, i.e. at the point indicated by the open circle. This spontaneous nodal discharge should occur before the following sinus impulse (S3) and thereby prevent the second capture. Thus, a second capture can only occur if the ectopic interval becomes temporarily longer than the sinus interval (considered at the level of the ectopic pacemaker). There are two mechanisms by which this may happen, either or both of which may be operative.

Mechanism 1: The Effect of Concealed Retrograde Conduction. If there is retrograde penetration of the nodal impulse, the first capturing impulse is delayed in the upper part of the A-V node (Diagram II of Fig. 6). The sinus interval is, therefore, not maintained to the level of the nodal pacemaker (the interval $\mathrm{X}-\mathrm{Y}$ is less than the interval S2-S3). The interval between sinus impulses may 
become less than the interval between ectopic impulses, even though the sinus rate is unchanged. This allows the second sinus impulse to anticipate the nodal impulse and be conducted through to the ventricles. This mechanism is always operative when consecutive paired captures occur during dissociation between sinus and ventricular pacemakers.

Mechanism 2: Transient Pacemaker Depression following Premature Discharge. A cardiac pacemaker may be depressed if it is prematurely discharged, e.g. by an extrasystole (Pick, Langendorf, and Katz, 1951). The more premature the discharge, the more marked will the subsequent depression be. In Diagram I of Fig. 6, the sinus impulse of the first capture beat discharges the nodal pacemaker prematurely at point $X$. If the subsequent depression of the nodal pacemaker is sufficiently marked, the next spontaneous nodal discharge will be postponed so that the nodal cycle becomes longer than the sinus cycle, and the second capture can then occur. This is the only mechanism allowing paired capture beats if there is no retrograde penetration of the nodal impulse. The amount of such depression of an active ectopic rhythm, however, is usually small and does not always occur. Therefore, if delay occurs in the lower zone only, it is highly unlikely that the second capture could occur. The first mechanism allowing paired consecutive captures is therefore the more likely one, and the very presence of consecutive captures is thus strong presumptive evidence of retrograde penetration of the nodal impulse.

Thus, during dissociation between sinus and A-V nodal pacemakers, partial retrograde penetration of the nodal impulse is inferred when paired consecutive captures are present; retrograde penetration is definitely present when the recovery line forms an angle greater than $\mathbf{4 5}$ degrees with the $\mathbf{X}$ axis, i.e. when the second capture interval is shorter than the first.

\section{SUMMARY}

Electrocardiograms showing A-V dissociation between consecutive capture beats are analysed. Concealed retrograde penetration of the A-V node by the ectopic pacemaker is invoked to explain apparent paradoxes. The effects of this assumption are explored. The mechanisms allowing consecutive ventricular captures are described.

This study was assisted by a grant to one of us (L.S.) by the Ernest Oppenheimer Memorial Trust, to whose Trustees sincere thanks are expressed. We are indebted to the Photo Unit, Dept. of Medicine, University of the Witwatersrand, for the photographic reproductions.

\section{REFERENCES}

Jedlicka, J. (1960). Die Erholungskurve der A-V-Leitungsfähigkeit. In Proceedings of the IIIrd European Congress of Cardiology, Rome, 1960, pp. 821-826. Excerpta Medica, Amsterdam.

Moe, G. K., Abildskov, J. A., and Mendez, C. (1964). An experimental study of concealed conduction. Amer. Heart J., 67, 338.

Pick, A., Langendorf, R., and Katz, L. N. (1951). Depression of cardiac pacemakers by premature impulses. Amer. Heart J., 41, 49, 115. 